\title{
Nutritional pathway for people with motor neurone disease
}

\begin{abstract}
This paper provides an overview of the nutritional management and care of people living with motor neurone disease (MND) in a specialist nutrition clinic. A specialist pathway of care has been developed to enable people living with MND to undergo a percutaneous endoscopic gastrostomy (PEG) procedure in a safe way; the pathway incorporates attendance at a dedicated nutrition clinic, a stratification tool to identify patients with a high periprocedural risk and a PEG insertion team with significant experience in the MND population. Since this pathway has been in place, gastrostomies have been successfully placed in patients with a forced vital capacity (FVC) of less than 50\%; previously, this would not have been possible.
\end{abstract}

\section{KEY WORDS}

$\square$ Motor neuron disease $\square$ hutrition $\square$ PEG $\square$ pathway

This article provides an overview of motor neuron disease (MND) and guidance for the management of patients with MND with regards to their nutritional status and nutritional support; it also aims to give an insight into how care is provided in a specialist MND care centre. We aim to give primary healthcare teams more confidence in their knowledge about caring for people with MND and on when and where to seek advice and information.

To discuss the nutritional pathway, it is first helpful to understand the nature of MND; it is a rare progressive neurological condition and few healthcare professions come in to contact with affected patients, which can make caring for these patients stressful. To date, there is no known cause or cure; however, a great deal can be done to help and support people living with the condition.

MND is characterised by a loss of upper and lower motor neurons and results in progressive muscle weakness and wasting. This causes difficulty with speech, swallowing, breathing and mobility. MND is a terminal disease with death usually attributed to respiratory failure. Fifty per cent of patients are alive within just 2-3 years of symptom onset (Talbot, 2009).

Symptoms of MND may begin in the limbs, the bulbar territory-corresponding to the muscles of speech and swallowing-or, more rarely, in the muscles used in respiration. Patients with bulbar onset MND comprise around $20 \%$ of cases, 
are typically older at onset and have shorter survival on average than those with limb onset (Talbot, 2009). The nature of MND is progressive, with symptoms spreading from the onset site to other areas of the body; this means that, over time, patients whose symptoms start in the limbs may develop respiratory and bulbar involvement, while those with bulbar involvement often develop problems with breathing, mobility and hand function (Talbot, 2009). The incidence is approximately 2 in 100000 (Motor Neurone Disease Association, 2016) of the population per year. Symptoms are most likely to first appear in people aged $55-75$ years. Approximately $10 \%$ of patients are younger than 45 years and $20 \%$ are aged over 70 years (Talbot and Marsden, 2008). The prevalence of MND is approximately 7 in 100000 . However, because of its rarity, it is thought that a GP may see one case of MND only every 10 years (Talbot, 2009).

Malnutrition is present in approximately $20 \%$ of patients with MND at diagnosis, irrespective of the presence of swallowing difficulties (Worwood and Leigh, 1998). Swallowing problems affect about $70 \%$ of people living with MND and increase the risk of malnutrition. Furthermore, difficulty in clearing saliva (1.5$2 \mathrm{~L}$ per day), nasal and respiratory secretions causes significant distress in patients with bulbar weakness.

Progression of MND is associated with declining muscle and adipose tissue reserves and there is evidence suggesting a $5 \%$ decrease in body weight is associated with a $30 \%$ increase in risk of earlier death (Marin et al, 2011). The high prevalence of malnutrition and the detrimental effects it can have demonstrate the importance of providing nutritional support within this patient group.

Reduced weight is an important indicator of poor nutrition (National Institute for Health and Care Excellence (NICE), 2016). People living with MND should undergo frequent monitoring of nutritional status, with ongoing advice and suggestions to modify diet and fluid consistency.

Nutritional supplementation via gastrostomy should be discussed with patients at an early stage before weight loss or respiratory insufficiency become an issue where possible, as there is evidence to suggest that early intervention leads to a better outcome and recovery (Forbes et al, 2004). In practice, this means that there is a 'window of opportunity' to perform a gastrostomy safely. The decision to have a gastrostomy is a complex, multifaceted process, which requires a multidisciplinary team approach (NICE, 2016).

Feeding via a nasogastric tube could be considered if a gastrostomy has been refused or is not possible (Katzberg and Benatar, 2011). However, supporting people at home is challenging, as community teams may have reservations 
about care and management.

Gastrostomy insertion is typically performed via endoscopy (percutaneous endoscopic gastrostomy (PEG) or radiological guidance (radiologically inserted gastrostomy, (RIG). Early data on gastrostomy insertion suggested that PEG insertion in patients with significant respiratory compromise were at higher risk of death within 30 days of the procedure; this led to a growth in the use of RIG (Kasarskis et al, 1999). However, more recent data from the gastrostomy in patients with amyotrophic lateral sclerosis study (ProGAS) has suggested no survival difference between the two gastrostomy insertion methods, with mortality within 30 days of the procedure at 3\% (ProGAS Study Group, 2015). There are additional considerations regarding the choice of insertion method including patient factors and local expertise.

Consequently, whether centres offer PEG or RIG tends to depend upon local expertise and experience. In Oxford, through close collaboration between the MND, endoscopy and respiratory teams, we have developed a pathway for PEG insertion. This incorporates attendance at a dedicated nutrition clinic, a stratification tool to identify patients at higher periprocedural risk, and a PEG insertion team with significant experience in the MND population.

\section{Nutrition clinic}

The nutrition clinic is run by a multidisciplinary team comprising the MND specialist nurse, nutrition specialist nurse, neurosciences dietitian and speech and language therapist. We have well-established multidisciplinary teamworking, which enables us to assess the patient's nutritional status holistically and make joint decisions regarding nutritional care. Our combined assessments identify the patient's current respiratory function, stage of disease, nutritional status and quality of life. We use a checklist as a prompt to ensure that all relevant information is collected and recorded.

Patients are referred to the MND nutrition clinic following identification of nutritional risk such as a bulbar onset diagnosis, concerns raised by the patient or those close to them, significant weight loss and/or difficulty swallowing. The time of referral to nutrition clinic varies, but patients are encouraged to attend as early as possible. Choosing the best time to introduce gastrostomy can be difficult. Patients with a new diagnosis, rapidly progressing disease or late diagnosis pose particular challenges as often assessment cannot be delayed. Patients are encouraged to bring supportive family members, friends or carers to guide them in making decisions.

The nutrition clinic provides patients with enough information for them to be able to make an informed decision regarding gastrostomy placement. Holding a compassionate discussion is integral to the nutrition clinic, but we strive to 
run the clinic from a neutral perspective. We address any barriers to gastrostomy if we are able to, but take care to allow patients to make a decision that is right for them.

A dietary recall is used to establish the type, amount, texture of foods eaten, along with any dietary restrictions (self-imposed or otherwise). While providing a basis on which the nutritional adequacy of a patient's diet can be assessed, the importance of eating and drinking for that patient can also assessed. Eating and drinking often become progressively less of a pleasure and more of a chore for patients with MND. Dietary advice is provided by the dietitian. This usually first involves food fortification advice and may include the provision of nutritional supplements. Patients are discouraged from unnecessary food restrictions and from following poorly evidenced diets that claim to be beneficial in the treatment of MND (ProGAS Study Group, 2015). If difficulties or concerns regarding swallowing are identified, a referral is made to speech and language therapy if the patient is not already known to the service. We are working towards establishing speech and language therapy input within the nutrition clinic following the release of the MND NICE guidelines (NICE, 2016).

Constipation is a common complaint, owing to reduced physical activity, and reduced fibre and fluid intake in MND patients. Swallowing difficulties, increased need for help to use the toilet, abdominal muscle weakness and constipating medications contribute to this. The adequacy of a patient's fluid intake is assessed to identify bowel function and also serves to indicate hydration status.

If the patient is happy and comfortable to proceed with a discussion about PEG, it is introduced delicately. We reiterate that our aim is to provide patients with enough information for them to be able to determine whether gastrostomy is a suitable option. We explain that gastrostomies unfortunately do not enable patients to live longer and the emphasis is instead placed on improving their quality of life (ProGas Study Group, 2015).

A model gastrostomy tube is shown to the patient and the flushing technique is demonstrated by the enteral nutrition nurse. The procedure for gastrostomy placement is explained and the medications involved are discussed. The risks, including infection, bleeding, diarrhoea, cramping, nausea and vomiting, oesophageal perforation and injury to the bowel, are outlined (Mitchell et al, 2008). It is highlighted to the patient that procedural complications are few and sedation can be used safely with early placement.

Depending on the outcome of the nutritional assessment, an indication as to how the patient might use the gastrostomy is provided and discussed. The options of bolus feeding, continuous pump feeding or a combination of the 
two are explained and the advantages and disadvantages of each discussed. Patients are often concerned that gastrostomy placement means that they will not be able to eat and drink orally but we reassure them that, if they wish to continue to eat and drink, they can do. However, the emphasis would be on the food and drink they enjoy. Nutrition support provided via a gastrostomy should reduce the pressure and effort that MND patients can feel to eat and drink sufficiently.

Care of the gastrostomy must be considered during nutrition clinic appointments to prevent delays to discharge once patients have been admitted. We assess patients' dexterity by asking them: 'Are you able to undo/do up buttons?' The patient is given opportunities to practise opening and closing the end of the gastrostomy tube and attaching a syringe to it. This allows us to determine how they might manage to do this independently on discharge. This is an important part of the planning process as it can highlight whether external care agencies need to be involved. Before discharge, training on gastrostomy care is given on the ward to the patient, family members and the patient's existing carers, such as care agency staff.

We explore the patient's wishes relating to resuscitation and ensure they are aware of their right to refuse this.

The overall risk of gastrostomy placement is determined according to a traffic light system (Box 1), whereby green indicates low risk, amber indicates moderate risk and red indicates high risk, with procedural modifications introduced in patients stratified amber or red. This method allows us to assess each patient as an individual and ensure the correct precautions will be taken at the time of the procedure to ensure it is carried out safely. It also enables us to have an open dialogue with the patient and their family about potential risks to them of going ahead with advanced respiratory muscle weakness, including death.

For example, patients identified as having a red or amber risk must be placed on an experienced consultant endoscopy list and gastrostomy insertion carried out by a team experienced in the care of patients with potential respiratory compromise. The procedure is done with the patients in a semirecumbent position of approximately 450 . For patients stratified red, noninvasive ventilation must be available before, during and after the procedure via a nasal mask, as patients need it.

Since this system has been in place, we have successfully placed gastrostomies in patients with a forced vital capacity (FVC) of $<50 \%$, who may otherwise not have undergone the procedure. Between January 2012 and August 2015, more than 155 people were seen in the nutrition clinic. Of these, 87 underwent gastrostomy insertion successfully with a mortality rate of $4 \%$ over 
the whole time frame of this study, comparable to that seen in the ProGAS research, despite $27 \%$ of patients having FVC of $<50 \%$ predicted before insertion (ProGAS Study Group, 2015).

\section{Decision making}

Some patients choose not to have a gastrostomy. In these cases, it is important to explore their rationale for refusal. While reasons vary, it is essential that any misconceptions are addressed, including taking into account apprehensions and anxieties about changing body image and sexuality as a result of the gastrostomy.

Patients may feel under pressure to have a gastrostomy. To reduce this, it is suggested that they take enough time to consider all options. Patients are asked to contact us once they have made a decision. However, if they do not respond, this is followed up at the next routine clinic appointment. If, ultimately, they choose not to have a PEG, then a plan for managing their deteriorating nutritional intake and support is then discussed and followed up by the dietitian and in the clinic.

For those who find making the decision difficult, we suggest the question is broken down into two:

Are you ever going to have a gastrostomy (yes or no)?

If yes, when?

\section{Timing of the gastrostomy}

As a team, we are keen to be as active as the patient will allow, because we have to consider their respiratory function, as well as their nutritional status and swallowing ability. For patients with bulbar MND who would ultimately wish to use a gastrostomy, early placement is preferred where feasible, since the procedure is safer before the development of respiratory muscle weakness. Our experience shows no adverse consequences of a gastronomy being in place in advance of regular use, so long as it is cared for in the usual way.

In our experience, patients often wish to delay gastrostomy insertion so a compromise must be reached. We negotiate a date that is suitable for the patient, depending on availability of team required according to the traffic light risk. If the date of insertion is more than 3 months from traffic light assessment or there has been significant deterioration, the traffic light assessment is repeated.

\section{Medications}

Swallowing medications in tablet form can be difficult for patients with MND, 
although some patients find ways to do this them more easily, including taking them in yoghurt or jam, or crushing them. Patients are advised that most medications can be safely administered enterally. We consult with pharmacy staff regarding the best form. Riluzole has recently been released in a liquid preparation called Teglutik, which can be used off label via gastrostomies.

\section{Discharge and follow up}

The role of the enteral homecare companies is explained to patients, including the delivery service of home equipment and supplies that the patient requires. We explain that enteral feeding nurses in the community can visit them at home should any problems arise during office hours (depending on their availability), and there is a 24-hour telephone helpline for out of hours. Follow up care is provided by the home enteral feeding dietitians. The MND team will continue to monitor patients at regular 2-3 monthly intervals.

\section{Conclusion}

The introduction of the nutrition clinic has enabled us to systematically review and assess patients living with MND who are experiencing difficulty eating and weight loss. We can assess the patient's nutritional status holistically and make decisions regarding nutritional care jointly. Using traffic light stratification, we can categorise patients according to potential risk and modify the way the PEG procedure is carried out.

\section{BJCN}

\section{KEY POINTS}

Motor neuron disease (MND) is a progressive neurological condition; because it is rare, few healthcare professionals come in to contact with affected patients

Around one in five patients with MND experience malnutrition, regardless of whether they have swallowing difficulties

Eating and drinking can become a chore for patients with MND and gastrostomies can improve quality of life

Nutritional supplementation via gastrostomy should be discussed with patients at an early stage

A specialist pathway enables people with MND to undergo percutaneous endoscopic gastrostomy safely; it has led to gastrostomies being successfully placed in patients with a forced vital capacity (FVC) $<50 \%$, which had not be possible before Patients may feel under pressure to have a gastrostomy, so should be given enough time to consider all options 
Accepted for publication: June 2016

Declarations of interest: none declared.

Forbes RB, Colville S, Swingler RJ; Scottish Motor Neurone Disease Research Group (2004) Frequency, timing and outcome of gastrostomy tubes for amyotrophic lateral sclerosis/motor neurone disease-a record linkage study from the Scottish Motor Neurone Disease Register. J Neurol 251(7): 813-7 Kasarskis EJ, Scarlata D, Hill R, Fuller C, Stambler N, Cedarbaum JM (1999) A retrospective study of percutaneous endoscopic gastrostomy in ALS patients during the BDNF and CNTF trials. J Neurol Sci 169(1-2): 118-25

Katzberg HD, Benatar M (2011) Enteral tube feeding for amyotrophic lateral sclerosis/motor neuron disease. Cochrane Database Syst Rev (1): CD004030.

Marin B, Desport JC, Kajeu P et al (2011) Alteration of nutritional status at diagnosis is a prognostic factor for survival of amyotrophic lateral sclerosis patients. J Neurol Neurosurg Psychiatry 82(6): 628-34

Mitchell SL, Tetroe JM, O'Connor AM, Rostom A, Villeneuve C, Hall B (2008) Making Choices: Long Term Feeding Tube Placement in Elderly Patients. Ottawa Hospital Research Institute, Canada. http://bestendings.wikispaces.com/file/view/TubeFeeding.pdf (accessed 16 June 2016) Motor Neurone Disease Association (2016) Motor Neurone Disease: a Problem Solving Approach. MND Association, London

National Institute for Health and Care Excellence (2016) Motor Neurone Disease: Assessment and Management. NICE Guidelines [NG42]. www.nice.org.uk/guidance/ng42 (accessed 16 June 2016)

ProGas Study Group (2015) Gastrostomy in patients with amyotrophic lateralsclerosis (ProGas): a prospective cohort study. Lancet Neurol 14(7): 702-9 Talbot K, Marsden R (2008) Motor Neuron Disease. The Facts. Oxford University Press, Oxford

Talbot K (2009) Motor neuron disease: the bare essentials. Pract Neurol 9(5): 303-9

Worwood AM, Leigh PN (1998) Indicators and prevalence of malnutrition in motor neurone disease. Eur Neurol 40(3): 159-63 


\section{Respiratory assessments where information is available}

\begin{tabular}{|c|c|c|c|}
\hline $\begin{array}{l}\text { Vital capacity (VC) } \\
\text { sitting/standing }\end{array}$ & $\ldots \mathrm{L} \_$\% pred & \multicolumn{2}{|c|}{$\begin{array}{l}\mathrm{ABC} / \mathrm{CBC} \\
\text { (delete as appropriate) }\end{array}$} \\
\hline VC lying (if done) & $\ldots \mathrm{L}$ & $\mathrm{pH}$ & - \\
\hline Fall in VC on lying & $\ldots$ L__ $\%$ fall & $\mathrm{PaCO}_{2}$ & $\ldots \mathrm{kPa}$ \\
\hline Sniff nasal pressure & $-\mathrm{cmH}_{2} \mathrm{O}$ & $\mathrm{PaO}_{2}$ & $\ldots \mathrm{kPa}$ \\
\hline \multirow[t]{2}{*}{$\mathrm{SaO}_{2}$ on pulseox } & _\% & Bicarbonate & $\ldots \mathrm{mM} / \mathrm{L}$ \\
\hline & & $\mathrm{SaO}_{2}$ & $\%$ \\
\hline
\end{tabular}

Please assess respiratory function as follows:

- Can the patient lie flat for 20 minutes? Yes/no

- Is their vital capacity greater than $50 \%$ predicted? Yes/no

- Does vital capacity fall by $15 \%$ or less on lying flat? Yes/no

- Is sniff nasal pressure $40 \mathrm{cmH}_{2} \mathrm{O}$ or greater? Yes/no

- Are arterial oxygen saturations $94 \%$ of greater? Yes/no

- On a blood gas, are $\mathrm{PaCO}_{2}<6 \mathrm{kPa}$ and bicarbonate $<27 \mathrm{mM} / \mathrm{L}$ ? Yes/no

\begin{tabular}{|l|l|l|l|}
\hline Risk & $\begin{array}{l}\text { Green light } \\
\text { (low risk) }\end{array}$ & $\begin{array}{l}\text { Amber light } \\
\text { (moderate risk) }\end{array}$ & $\begin{array}{l}\text { Red light } \\
\text { (high risk) }\end{array}$ \\
\hline Criteria & $\begin{array}{l}\text { Answers 'yes' } \\
\text { to all questions } \\
\text { above }\end{array}$ & $\begin{array}{l}\text { Answer to one } \\
\text { or more quetions } \\
\text { above is 'no' } \\
\text { Pac } \text { CO }_{2} \text { or } \\
\text { bicarbonate raised } \\
\text { Patient is already } \\
\text { on NIV } \\
\text { Patient cannot lie } \\
\text { flat }\end{array}$ \\
\hline Action & $\begin{array}{l}\begin{array}{l}\text { PEG can be } \\
\text { carried out on } \\
\text { a routine list } \\
\text { (re-evaluate if } \\
\text { delay between } \\
\text { referral and } \\
\text { procedure is } \\
>2 / 3 \text { months) }\end{array} \\
\begin{array}{l}\text { PEG must be } \\
\text { carried out on } \\
\text { a consultant } \\
\text { list by a team } \\
\text { experienced in the } \\
\text { care of patients } \\
\text { with potential } \\
\text { respiratory } \\
\text { complications }\end{array}\end{array}$ & $\begin{array}{l}\text { As for amber, but NIV } \\
\text { must be available for } \\
\text { use before, after and } \\
\text { during the procedure } \\
\text { via a nasal mask }\end{array}$ \\
\hline
\end{tabular}

